\title{
Photoredox-Catalyzed Reduction of Halogenated Arenes in Water by Amphiphilic Polymeric Nanoparticles
}

\author{
Fabian Eisenreich, Tom H. R. Kuster, David van Krimpen and Anja R. A. Palmans *
}

Citation: Eisenreich, F.; Kuster, T.H.R.; van Krimpen, D.; Palmans, A.R.A. Photoredox-Catalyzed Reduction of Halogenated Arenes in Water by Amphiphilic Polymeric Nanoparticles. Molecules 2021, 26 5882. https://doi.org/10.3390/ molecules26195882

Academic Editor: Cyrille Boyer

Received: 3 September 2021

Accepted: 24 September 2021

Published: 28 September 2021

Publisher's Note: MDPI stays neutral with regard to jurisdictional claims in published maps and institutional affiliations.

Copyright: (c) 2021 by the authors. Licensee MDPI, Basel, Switzerland. This article is an open access article distributed under the terms and conditions of the Creative Commons Attribution (CC BY) license (https:/ / creativecommons.org/licenses/by/ $4.0 /)$.
Laboratory of Macromolecular and Organic Chemistry, Institute for Complex Molecular Systems, Eindhoven University of Technology, P.O. Box 513, 5600 MB Eindhoven, The Netherlands; f.r.eisenreich@tue.nl (F.E.); t.h.r.kuster@student.tue.nl (T.H.R.K.); d.v.krimpen@student.tue.nl (D.v.K.)

* Correspondence: a.palmans@tue.nl

\begin{abstract}
The use of organic photoredox catalysts provides new ways to perform metal-free reactions controlled by light. While these reactions are usually performed in organic media, the application of these catalysts at ambient temperatures in aqueous media is of considerable interest. We here compare the activity of two established organic photoredox catalysts, one based on 10-phenylphenothiazine (PTH) and one based on an acridinium dye (ACR), in the light-activated dehalogenation of aromatic halides in pure water. Both PTH and ACR were covalently attached to amphiphilic polymers that are designed to form polymeric nanoparticles with hydrodynamic diameter $\mathrm{D}_{H}$ ranging between 5 and $11 \mathrm{~nm}$ in aqueous solution. Due to the hydrophobic side groups that furnish the interior of these nanoparticles after hydrophobic collapse, water-insoluble reagents can gather within the nanoparticles at high local catalyst and substrate concentrations. We evaluated six different amphiphilic polymeric nanoparticles to assess the effect of polymer length, catalyst loading and nature of the catalyst (PTH or ACR) in the dechlorination of a range of aromatic chlorides. In addition, we investigate the selectivity of both catalysts for reducing different types of aryl-halogen bonds present in one molecule, as well as the activity of the catalysts for $\mathrm{C}-\mathrm{C}$ cross-coupling reactions. We find that all polymer-based catalysts show high activity for the reduction of electron-poor aromatic compounds. For electron-rich compounds, the ACR-based catalyst is more effective than PTH. In the selective dehalogenation reactions, the order of bond stability is $\mathrm{C}-\mathrm{Cl}>\mathrm{C}-\mathrm{Br}>\mathrm{C}$-I irrespective of the catalyst applied. All in all, both water-compatible systems show good activity in water, with ACR-based catalysts being slightly more efficient for more resilient substrates.
\end{abstract}

Keywords: amphiphilic copolymer; photoredox catalysis; polymer assembly; hydrophobic collapse; compartmentalization; dehalogenation

\section{Introduction}

Photoredox catalysis has emerged in the last decade as a powerful tool for chemical bond transformations and late-stage functionalization of pharmaceutically relevant compounds [1-5]. Since reactions only occur when the catalyst is in its excited state, which can be accurately controlled by light, photoredox-catalyzed reactions show an unprecedentedly high degree of spatial and temporal control. Traditionally, photoredox-catalyzed reactions have been performed in organic solvents, as this permits good mixing between the catalyst, the substrates and the products. More recently, photoredox catalysis has also become feasible in water by using micellar systems, water compatible ligands, or water-soluble counter ions for the metal-based catalysts [6-10]. Water is considered a green solvent, making reactions more environmentally benign. At the same time, water also facilitates recyclability of the catalysts, which is of high interest when expensive transition metals are applied $[6,8]$.

Organocatalytic alternatives for photoredox catalysts have been developed to avoid the use of expensive Ir- and Ru-based systems and to tune the redox potential of the catalysts [5,11-13]. Their synthetic potential has been illustrated by their applicability for a 
broad spectrum of substrates and in a range of reactions such as polymerizations, substitutions, bond cleavage reactions and cycloadditions [2,14-16]. Usually, these organocatalysts are evaluated in organic media, making separation of products, solvent and catalyst laborious. Additionally, here, the use of water as solvent by introducing micellar systems [17-21] or by using water-soluble catalytic complexes [22,23] have shown great potential. Micellar systems are especially interesting since the hydrophobic domains present in the aqueous medium allow us to increase the local catalyst concentration, and when hydrophobic substrates are used this results in faster conversion at equal catalyst loading [24,25]. However, if the catalyst is not covalently bound, it can exit the hydrophobic domains. To prevent the catalyst from leaking out, covalent linking of the catalyst to a polymer backbone is a promising way to retain high catalyst activity and stability [26-28]. We recently observed that covalently attaching the highly reductive 10-phenylphenothiazine catalysts to an amphiphilic polymer resulted in stable and highly active photoredox catalysts in water. In addition, we observed that the polymer catalysts could be reused without loss of activity up to five times [23].

In this work, we present a systematic investigation on the reactivity of two organic photoredox catalysts namely 10-phenylphenothiazine (PTH) [14,29] and the acridinium dye 9-mesityl-3,6-di-tert-butyl-10-phenyl-acridinium (ACR) [16] covalently attached to watersoluble polymeric nanoparticles (PNs) (Scheme 1). The PNs permit the catalyst to be taken up in the hydrophobic pocket [30-33], which together with accumulation of hydrophobic substrates affords a high local concentration of both the catalyst and the substrate. The acridinium catalyst $\left(\mathrm{E}_{1 / 2}{ }^{*}=-3.36 \mathrm{~V}\right.$ vs. SCE) is an exceptionally strong reductant in its excited state but needs to be excited twice before the desired excited state is reached [16]. The PTH catalyst $\left(\mathrm{E}_{1 / 2}{ }^{*}=-2.1 \mathrm{~V}\right.$ vs. SCE $)$ is also a very strong reductant but less potent when compared to the ACR. It does, however, require one excitation only to reach the desired excited state [14,15]. For PTH, dehalogenation reactions [14,15,23], C-C crosscoupling reactions $[15,23]$ and radical polymerizations $[29,34]$ have been reported. ACR catalysts have also been investigated in dehalogenations [35], cross-coupling reactions [36], halogenations [36] and radical polymerizations [37]. We here select the dehalogenation of aryl chlorides as a benchmark reaction to compare the two systems in water and to elucidate how far the degree of polymerization, catalyst loading, and the nature of the catalyst, PTH or ACR, affect the reactivity towards dechlorination reactions. In addition, we investigate in how far selectivity can be attained in dehalogenations of different carbonhalogen bonds. Finally, we evaluate the feasibility of a cross-coupling reaction of an aryl chloride with $N$-methylpyrrole.

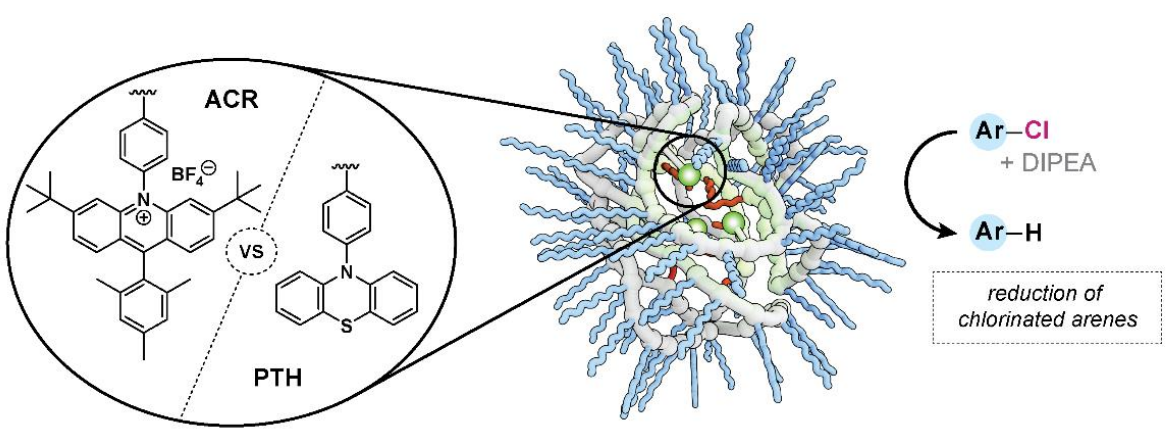

Scheme 1. Schematic representation of the PNs formed in water, the chemical structures of the photoredox catalysts and the dichlorination reaction of aromatic chlorides used in this work.

\section{Methodology and Results}

\subsection{Molecular Design, Synthesis, and Characterization of Photocatalyst Loaded PNs}

We designed and synthesized six different amphiphilic polymers, PN1-6, with the aim of assessing the influence of the polymer chain length, catalyst loading and catalyst type on catalyst activity in aqueous solutions (Scheme 2). To this end, we first synthesized two polyacrylates ( $\mathbf{P}_{\mathbf{1 0 0}}$ and $\mathbf{P}_{\mathbf{2 0 0}}$ ) with degrees of polymerization (DP) of 100 and 200, using 
pentafluorophenyl acrylate as the monomer [38]. The two polyacrylates were made by reversible addition-fragmentation chain-transfer (RAFT) polymerization of the acrylate, followed by the removal of the RAFT end group [39]. The DP was determined by monitoring the monomer conversion with ${ }^{19} \mathrm{~F}$ NMR spectroscopy. The molar mass distributions $(\nexists)$ of the polymers were narrow (e.g., $\left.Ð\left(\mathbf{P}_{\mathbf{2 0 0}}\right)=1.15\right)$ as evidenced by size-exclusion chromatography (SEC), calibrated by polystyrene standard in THF, indicating the controlled nature of the polymerization (Figure S1 in the Supporting Information).
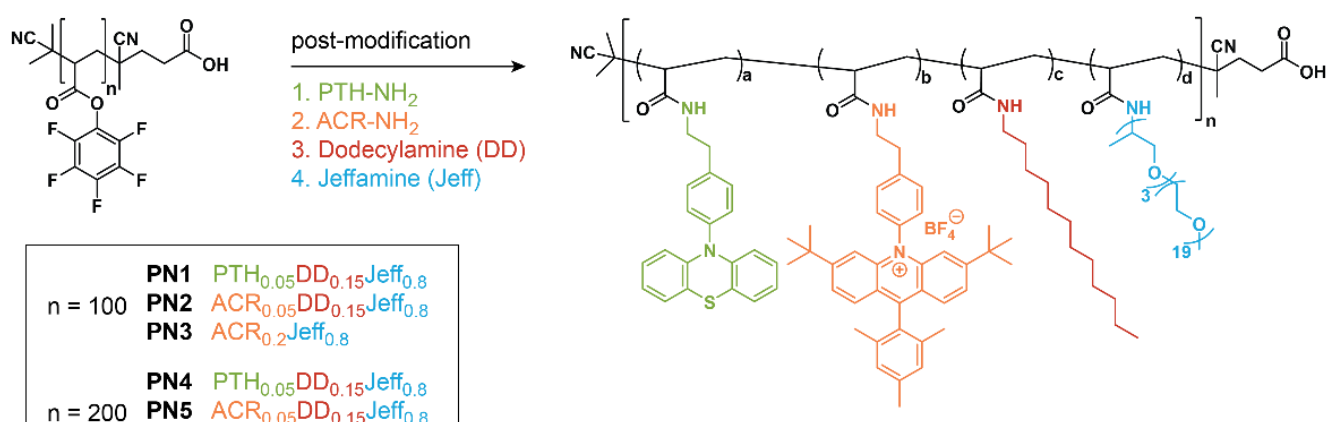

$\mathrm{n}=200$ PN5 ACR $0.05 \mathrm{DD}_{0.15} \mathrm{Jeff}_{0.8}$

PN6 ACR 0.2 Jeff $_{0.8}$

Scheme 2. Stepwise post-functionalization of polyacrylate prepolymer affords PN1-PN6.

In order to covalently attach the selected photoredox catalysts, PTH and ACR, to the polymer backbone, both catalysts were equipped with an amine functional group. PTH$\mathbf{N H}_{\mathbf{2}}$ was prepared according to our previously reported procedure [23] whereas ACR$\mathbf{N H}_{\mathbf{2}}$ was synthesized following a modification of the procedure reported for ACR [35]. By changing the aniline used in the final synthesis step to BOC-protected 4-(aminoethyl)aniline, followed by removal of the BOC group, the desired ACR- $\mathbf{N H}_{2}$ was readily accessed. The details of the ACR- $\mathbf{N H}_{2}$ synthesis and its molecular characterization are given in the Supplementary Information.

After successfully acquiring both amine-functional catalysts, the reactive ester side groups of the poly(pentafluorophenyl) acrylates were sequentially substituted by the desired PTH- $\mathbf{N H}_{\mathbf{2}}(5 \%)$ or ACR- $\mathbf{N H}_{2}$ ( 5 or $20 \%$ ), followed by hydrophobic $n$-dodecylamine (15 or $0 \%$ ), and hydrophilic Jeffamine@1000 (80\%) (Scheme 2). We added $n$-dodecylamine as additional hydrophobic moiety as it enhances the hydrophobic collapse of the polymers in water [40]. A random distribution of the hydrophobic/hydrophilic moieties along the polymer chain is important as this helps to induce an intramolecular, hydrophobic collapse of the amphiphilic copolymer in water [41,42], which results in the formation of unimolecular polymeric nanoparticles of defined size. The conversion of each step when adding the amines sequentially was assessed by ${ }^{19} \mathrm{~F}$ NMR spectroscopy by comparing the sharp peaks of the released pentafluorophenol to the broad peaks of the polymer bound pentafluorophenyl group (Figures S2-S7 in the Supplementary Information). We synthesized six different, fully functionalized polymers (PN1-6). PNs with a DP of 100, PN1-3, have a theoretical molecular weight of 93-101 kDa and $Ð$ of 1.15-1.19. PNs with a DP of 200, PN4-6, have a theoretical molecular weight of 187-202 kDa and $Đ$ of 1.17-1.31. The molar mass distributions were determined by SEC, calibrated with poly(ethyleneoxide) in DMF with LiBr (full SEC traces are in the Supplementary Information Figure S8).

All polymers were well soluble in water. Their hydrodynamic diameters $\left(D_{\mathrm{H}}\right)$ in water were determined by dynamic light scattering (DLS) measurements (Figure S9 in the Supplementary Information). The DLS traces show unimodal size distributions, with $D_{\mathrm{H}}$ ranging between 5 and $11 \mathrm{~nm}$. These sizes are in line with those observed for systems of similar DP and microstructure, and indicate that all polymers, including those with $20 \%$ acridinium groups attached, form defined nanoparticles in water $[39,40]$. DLS, however, does not exclude that particles comprise multiple polymer chains. The larger sizes of some of the polymers (PN1, PN2 and PN5) may indicate some clustering of polymer chains. 
For the catalytic studies, however, this is not important, as long as the particles are of defined size.

Finally, the optical properties of the nanoparticles were studied with UV/Vis spectroscopy with water as solvent (Figure S10 in the Supplementary Information). It was previously reported that the PTH absorption maxima are located around 258 and $320 \mathrm{~nm}$ [43], whereas ACR absorption maxima occur at 360 and $520 \mathrm{~nm}$ [16]. Both the polymer bound ACR and PTH moiety show absorption bands identical to those reported in the literature for the free catalysts. This shows that both catalysts have been successfully attached to the PNs and that the optical properties are not affected by the presence of the amphiphilic polymer.

\subsection{Reduction of Para-Substituted Aryl Chlorides in Water Using PNs}

As a benchmark and to compare the reducing efficiency of our PNs to previously reported systems in organic media, we selected the dechlorination of aryl chlorides $[14,16,23,43,44]$. We selected five chlorinated benzene derivatives S1-S5 each with a different para-substituent, ranging from electron-donating (S2) to electron-withdrawing (S1, S3) or only exerting inductive effects (S4, S5) (Table 1). Regarding the choice of substrates, we did not include substrates with strong electron donors, such as dimethylamine groups, as they could potentially act as the proton or electron source and thus result in side reactions. Nitro compounds, as electron poor substrates, were initially tested but yielded many side products, as nitro substrates undergo photolysis reactions under light illumination [45]. All reactions were performed after degassing the solutions with argon in stirred glass vials, using $385 \mathrm{~nm}$ light-emitting diode (LED) light. Note that the presence of a tertiary amine base, such as $\mathrm{N}, \mathrm{N}$-diisopropylethylamine (DIPEA), is paramount for the catalytic cycle as it acts as a sacrificial proton and electron source [14,16]. Details of the reaction setup and the involved reaction mechanisms are given in the Supplementary Information (Figures S11-S13). The conversions were measured by NMR spectroscopy (Figures S14-S19) and the results of the dechlorinations are shown in Table 1.

Table 1. Conversion of para-substituted chlorobenzenes to benzene derivatives using PN1-6 ${ }^{1}$.

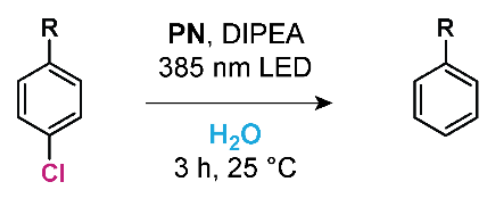

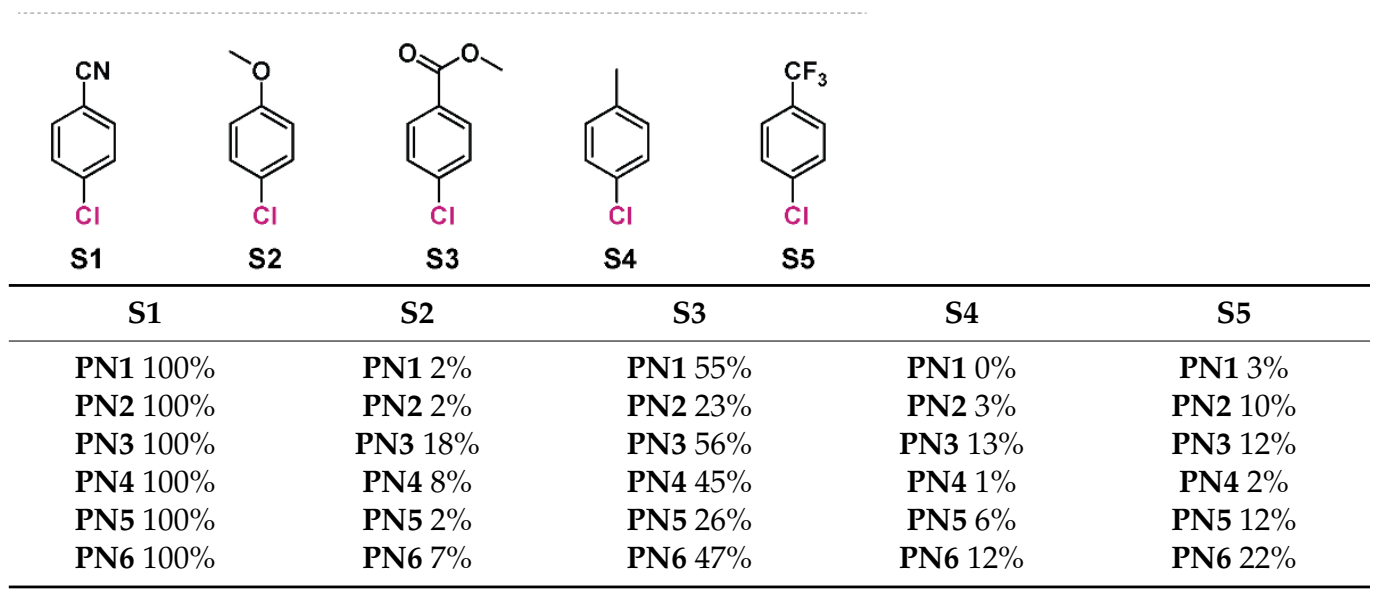

${ }^{1}$ For all reactions, $10 \mathrm{mg}$ PN was added to $0.5 \mathrm{~mL}$ of $\mathrm{H}_{2} \mathrm{O}$. $\mathrm{S} / \mathrm{C}=90: 1$ for PN1, PN2, PN4 and PN5; $\mathrm{S} / \mathrm{C}=25: 1$ for PN3 and PN6. $[\mathrm{S}]=0.1 \mathrm{M} ;[\mathrm{DIPEA}]=0.5 \mathrm{M}$. All reactions were conducted according to the general procedure for photoredox dehalogenation in water.

Electron poor aromatic systems are expected to show higher conversions compared to electron rich systems $[16,23]$. The results indeed show that electron poor $\mathbf{S} 1$ is completely reduced after $3 \mathrm{~h}$ for all PN1-6. With S3, the conversions are lower, between 23 and 55\%. Electron rich S2, in contrast, showed a significant decrease in conversion, which only 
ranged between 2 and 18\%. Additionally, methylated and trifluoromethylated S4 and S5, less electron poor than $\mathbf{S 1}$, show low conversions ranging between 0 and $22 \%$. Interestingly, the 5\% PTH-loaded polymers PN1 and PN4 showed a higher conversion than the 5\% ACR-loaded polymers PN2 and PN5. When looking at the more electron rich substrates 4-chlorotoluene S3 and 4-chlorobenzotrifluoride S4, the 5\% ACR-loaded polymers show higher conversion compared to the PTH-loaded analogues, indicating the importance of a higher reduction potential for these more resilient substrates. 4-Chloroanisole $\mathbf{S} 2$ showed higher conversion for both the PTH-loaded polymers, despite the fact that the methoxy group is an electron donating group. The influence of catalyst loading on conversion turned out as anticipated. The polymeric nanoparticles with 20\% ACR PN3 and PN6 outperformed the 5\% ACR polymers PN2 and PN5 for every substrate, which is likely related to the decrease in the S/C ratio from 90:1 to 25:1. No obvious correlation between the length of the polymeric backbone and conversion was established, indicating that the DP of the polymer is not relevant to tune catalyst activity.

All in all, PN3 and PN6 show the highest conversion for most of the used substrates. The activity of the PTH-loaded polymers PN1 and PN4 is high for less resilient substrates, but quickly drops in activity as the electron-withdrawing nature of the substituent decreases. Higher conversions can, however, be reached by irradiating the reaction mixtures for longer periods of time. Methyl 4-chlorobenzoate S3 showed almost full conversion $(90-98 \%)$ for all six polymers after $18 \mathrm{~h}$ of irradiation.

\subsection{Investigating Selectivity of Dehalogenation with PTH and ACR}

As shown above, different conversions for the dechlorinations were observed between ACR- and PTH-based PNs between electron poor and electron rich aromatic chlorides. A difference in conversion can also occur when different types of halogens are attached to the benzene ring, due to the different reduction potentials between $\mathrm{C}-\mathrm{Cl}, \mathrm{C}-\mathrm{Br}$ and C-I bonds [14-16]. The reduction potential decreases from $\mathrm{C}-\mathrm{Cl}\left(\mathrm{E}_{1 / 2}=-2.8 \mathrm{~V}\right.$ for chlorobenzene) [46] to C-I ( $\mathrm{E}_{1 / 2}=-1.6 \mathrm{~V}$ for iodobenzene) [47] bonds, making C-Cl the most resilient to reductive dehalogenation, and C-I the most reactive [48]. To assess whether the PNs in water with either PTH or ACR attached show selectivity towards reducing carbon-halogen bonds, benzenes with different carbon-halogen bonds were evaluated as substrates. As previously shown by Poelma et al. [15] in organic media, selective dehalogenation can be achieved by controlling the reaction time or using photoredox catalysts with different excited state reduction potentials.

To identify the difference in dehalogenation capacity of both photocatalysts, three substrates were selected to perform these selectivity reactions: 1-chloro-4-iodobenzene S6, 1-bromo-4-chlorobenzene S7 and 2-bromo-4-chloro-1-iodobenzene S8. The conversion of these reactions was determined via ${ }^{1} \mathrm{H}$ NMR spectroscopy (Figures S20-S24 in the Supplementary Information) and the results are summarized in Table 2.

The C-I bond in 1-chloro-4-iodobenzene $\mathbf{S} \mathbf{6}$ was preferentially reduced by all polymers, but some C-Cl was also reduced (between 1-8\%). Interestingly, the 20\% ACR- loaded polymers PN3 and PN6 showed the lowest conversion of S6 but the highest amount of benzene formation, indicating a lower activity and lower selectivity. The 5\% ACR-loaded polymers PN2 and PN5 showed similar conversions to the PTH-loaded polymers, but a slightly higher conversion towards benzene. This suggests that PTH-based PNs are slightly more selective in breaking C-I bonds in halogenated aromatic compounds, likely a result of their lower reducing ability.

1-Bromo-4-chlorobenzene, $\mathbf{S 7}$, showed, as expected, significantly lower conversions, ranging from 6 to $49 \%$, a result of the higher reduction potential of the C-Br bond. Especially the PTH-loaded polymers PN1 and PN4 showed a significant decrease in catalytic activity, indicating the importance of the strong reductive nature of the excited state of acridinium to reduce more resilient halogens. Additionally, here, only acridinium loaded polymers were able to reduce the halogenated substrate to benzene, with the two $20 \%$ acridinium loaded polymers, PN3 and PN6, forming the most benzene. 
Table 2. Conversions of substituted benzenes S6-S7 with functionalized polymers PN1-6 into benzene derivatives 1-chloro-4-iodobenzene $\mathbf{S} 6$ and 1-bromo-4-chlorobenzene $\mathbf{S 7}{ }^{1}$.

a)
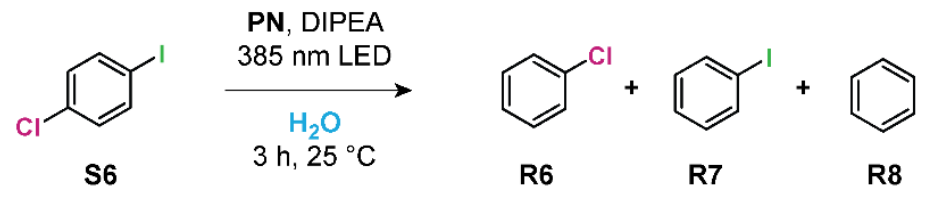

b)
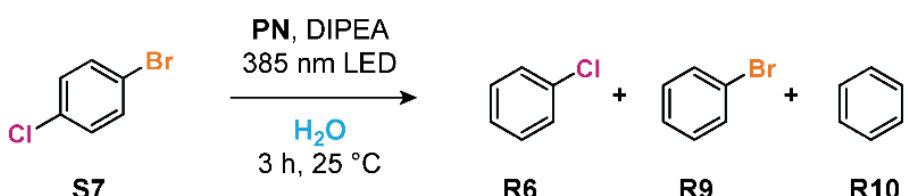

\begin{tabular}{|c|c|c|c|c|c|}
\hline \multicolumn{3}{|c|}{ Reaction a } & \multicolumn{3}{|c|}{ Reaction b } \\
\hline $\mathbf{R 6}$ & R7 & $\mathbf{R} 8$ & R6 & R9 & R8 \\
\hline PN1 65\% & PN1 0\% & PN1 2\% & PN1 6\% & PN1 6\% & PN1 $>1 \%$ \\
\hline PN2 68\% & PN2 0\% & PN2 4\% & PN2 13\% & PN2 13\% & PN2 4\% \\
\hline PN3 51\% & PN3 0\% & PN3 6\% & PN3 41\% & PN3 41\% & PN3 6\% \\
\hline PN4 63\% & PN4 0\% & PN4 1\% & PN4 8\% & PN4 8\% & $\mathbf{P N} 4>1 \%$ \\
\hline PN5 68\% & PN5 0\% & PN5 7\% & PN5 22\% & PN5 22\% & PN5 7\% \\
\hline PN6 38\% & PN6 0\% & PN6 8\% & PN6 19\% & PN6 19\% & PN6 8\% \\
\hline
\end{tabular}

${ }^{1}$ For all reactions, $10 \mathrm{mg}$ PN was added to $0.5 \mathrm{~mL}$ of $\mathrm{H}_{2} \mathrm{O}$. S/C = 90:1 for PN1, PN2, PN4 and PN5; S/C = 25:1 for PN3 and PN6. $[\mathrm{S}]=0.1 \mathrm{M} ;[\mathrm{DIPEA}]=0.5 \mathrm{M}$. All reactions were conducted according to the general procedure for photoredox dehalogenation in water.

Finally, 2-bromo-4-chloro-1-iodobenzene, S8, was reduced to identify which products are mainly formed. The major products were 1-bromo-3-chlorobenzene R10 and chlorobenzene $\mathbf{R 6}$, and neither bromobenzene $\mathbf{R} 9$ nor iodobenzene $\mathbf{R} 7$ were formed, indicating the clear preference for cleavage of carbon halogen bonds for halogens with lower reduction potentials. In none of the examples discussed above did we observe a difference in conversion between polymers with different degrees of polymerization, suggesting that polymer length does not play a notable role in the activity or selectivity of the catalysts.

\subsection{C-C Cross-Coupling Reaction with N-Methylpyrrole}

We explored the C-C cross-coupling reaction between 4-chlorobenzonitrile, S1, and $\mathrm{N}$-methylpyrrole, S9. 4-Chlorobenzonitrile $\mathbf{S 1}$ was selected as most suitable substrate as it showed full conversion after $3 \mathrm{~h}$ of irradiation for all six PNs. In this reaction, two product formations are competing, namely the formation of C-C cross-coupled R11 and reduced product R1 (Table 3, Figure S24). In order to shift the selectivity of this reaction to the C-C cross-coupled product, an excess of $N$-methylpyrrole (25-50 equivalents) is usually added $[15,46]$. We previously observed in the reaction of 2-iodobenzonitrile with $\mathrm{N}$ methylpyrrole that the high local concentration of $\mathrm{N}$-methylpyrrole within the hydrophobic compartments of nanoparticles led to an increase in the selectivity towards the C-C crosscoupled product, thus only five equivalents of $\mathrm{N}$-methylpyrrole were required [23]. Here, we find a selectivity towards the cross-coupled product R11 up to $48 \%$. Both the $5 \%$ PTHand 20\% ACR-loaded polymers PN1, PN3-4, and PN6 showed a similar selectivity of roughly 47\% towards R11. The 5\% ACR-loaded polymers PN2 and PN5, surprisingly, showed a lower selectivity towards the cross-coupled product of roughly $35 \%$. In all cases, the selectivity is somewhat lower than that previously reported for PTH-loaded systems, which showed a selectivity for the cross-coupled product of up to $78 \%$ using 2-iodobenzonitrile as the substrate. 
Table 3. C-C cross-coupling of 4-chlorobenzonitrile S1 and N-methylpyrrole S9. Full conversion of 4-chlorobenzonitrile was observed, forming both the C-C cross-coupled R11 and dehalogenated $\mathbf{R} \mathbf{1}$ product. The selectivity to both products is shown as ratio of $\mathbf{R 1 1}$ and $\mathbf{R} \mathbf{1}$. The reactions were prepared according to the general protocol discussed in the experimental section, with the addition of 5 equivalents of $N$-methylpyrrole $\mathbf{S 9}{ }^{1}$.

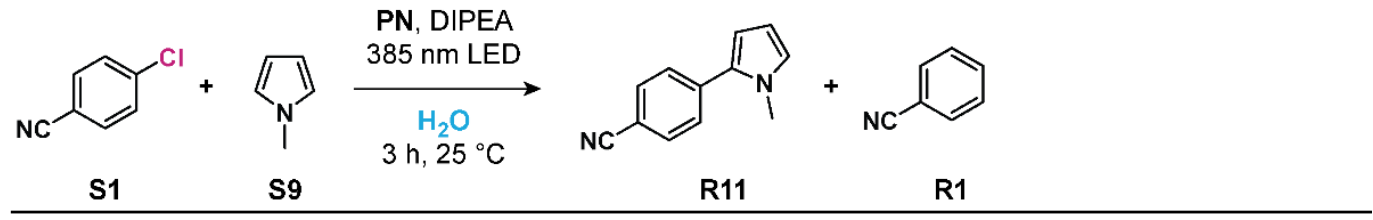

\begin{tabular}{ccc}
\hline PN & Conversion & Ratio R11:R1 \\
\hline PN1 & $100 \%$ & $46: 54$ \\
PN2 & $100 \%$ & $37: 63$ \\
PN3 & $100 \%$ & $47: 53$ \\
PN4 & $100 \%$ & $48: 52$ \\
PN5 & $100 \%$ & $33: 67$ \\
PN6 & $100 \%$ & $48: 52$ \\
\hline
\end{tabular}

${ }^{1}$ For all reactions, $10 \mathrm{mg}$ PN was added to $0.5 \mathrm{~mL}$ of $\mathrm{H}_{2} \mathrm{O}$. S/C $=90: 1$ for PN1, PN2, PN4 and PN5; $\mathrm{S} / \mathrm{C}=25: 1$ for PN3 and PN6. [S1] = 0.1 M; [DIPEA] = 0.5 M; [S9] = 0.5 M. All reactions were conducted according to the general procedure for photoredox dehalogenation in water.

\section{Discussion}

The two reductive organic photoredox catalysts, PTH and ACR, covalently attached to an amphiphilic polymeric backbone show promising results in the reduction of aryl chlorides in aqueous media. It has to be noticed, however, that conversions seem rather low, especially for the more resilient substrates. This is mainly related to the low catalyst concentrations we used ( 1 or $4 \mathrm{~mol} \%$ ) and the limited reaction time of $3 \mathrm{~h}$. When comparing the results found in this work to results previously reported, which is challenging as the conditions are never identical, we can make some interesting observations. PTH-loaded PNs are much more efficient in the reduction of 4-chlorobenzonitrile and methyl 4-chlorobenzoate in water than free PTH in acetonitrile: Using $5 \mathrm{~mol} \%$ of catalyst, Discekici et al. needed $72 \mathrm{~h}$ to achieve $92 \%$ conversion for both substrates [14], while in our case complete conversion was reached within $3 \mathrm{~h}$ for 4 -chlorobenzonitrile and $16 \mathrm{~h}$ for methyl 4-chlorobenzoate using only $1 \mathrm{~mol} \%$ of catalyst. Additionally, for ACR-loaded PNs the reduction of 4-chlorobenzonitrile in water is more efficient than in acetonitrile: Nicewicz et al. needed $16 \mathrm{~h}$ to achieve $78 \%$ conversion using $10 \mathrm{~mol} \%$ catalysts whereas we found full conversion in $3 \mathrm{~h}$ for $1 \mathrm{~mol} \%$ catalyst [16]. For the more demanding substrates, 4-chloromethoxybenzene and 4-chlorotrifluoromethylbenzene, Nicewicz et al. reported conversions of 82 and $58 \%$, respectively, after $16 \mathrm{~h}$ using $10 \mathrm{~mol} \%$ catalysts, whereas we found best conversions of 18 and $22 \%$, respectively, after $3 \mathrm{~h}$ with $4 \mathrm{~mol} \%$ catalyst [16]. It is likely that similar or even higher conversions will be attained when the reaction runs for $16 \mathrm{~h}$. All in all, the most noticeable difference between the different PNs reported in this work, is the lower selectivity that ACR-loaded PNs show in dehalogenations. Although the differences are not that significant, it is clear that PTH is the best catalyst when selectivity is desired. On the other hand, ACR-loaded PNs are active for more resilient substrates.

\section{Materials and Methods}

\subsection{Materials}

All solvents and chemicals used were of reagent grade quality and purchased from Biosolve (Valkenswaard, The Netherlands) or Sigma-Aldrich (Amsterdam, The Netherlands) at the highest purity available and used without further purification unless otherwise noted. Water for aqueous samples was purified on an EMD Millipore Milli-Q Integral Water Purification System (Merck, Amsterdam, The Netherlands). Methyl 2,4,6-trimethyl 
benzoate [49], 3,6-di-tert-butyl-9-mesitylxanthylium tetrafluoroborate [16], PTH-NH 2 [23], and $\mathbf{P}_{\mathbf{1 0 0}}$ [23] were prepared according to procedures detailed in the literature.

\subsection{Methods}

NMR measurements were performed on a Bruker $400 \mathrm{MHz}$ Ultrashield spectrometer (Karlsruhe, Germany) $\left({ }^{1} \mathrm{H}\right.$ at $400 \mathrm{MHz},{ }^{13} \mathrm{C}$ at $100 \mathrm{MHz}$ and ${ }^{19} \mathrm{~F}$ at $376 \mathrm{MHz}$ ) at $25{ }^{\circ} \mathrm{C}$. The compound of interest was dissolved in $\mathrm{CDCl}_{3}$, obtained from Cambridge Isotope Laboratories (Tewksbury, MA, USA), with the internal standard for ${ }^{1} \mathrm{H}$ and ${ }^{13} \mathrm{C} \delta_{\mathrm{CDCl}}=7.26 \mathrm{ppm}$ and $\delta_{\mathrm{CDCl} 3}=77.16 \mathrm{ppm}$, respectively. Multiplicities are indicated with the following abbreviations: singlet (s), doublet (d), doublet of doublet of doublets (ddd), triplet (t), quartet (q), multiplet (m), and broad (br). The obtained NMR spectra were processed using MestReNova v14.0.1-23559 (Mestrelab Research, Santiago de Compostela, Spain). MALDI-TOF-MS spectra were measured by a Bruker Autoflex Speed MALDI-TOF (Karlsruhe, Germany) using either $\alpha$-cyano-4-hydroxycinnamic acid (CHCA) or trans-2-[3-(4-tert-butylphenyl)2-methyl-2-propenylidene]malononitrile (DCTB) as matrix material. UV/VIS absorption spectra were measured on a JASCO V-650 spectrometer (de Meern, The Netherlands) with a JASCO CTU-100 Circulating Thermoset Unit at $20^{\circ} \mathrm{C}$. The compound of interest was dissolved in water $\left(c=0.4 \mathrm{mmol}_{\text {catalyst }} / \mathrm{L}\right.$ ). Dynamic Light Scattering (DLS) spectra were obtained from a Malvern $\mu \mathrm{V}$ Zetasizer (Etten-Leur, The Netherlands) with a $830 \mathrm{~nm}$ laser and scatter angle of $90^{\circ}$. Disposable UV-transparent cuvettes from Sarstedt (Nuembrecht, Germany) with a path length of $10 \times 2 \mathrm{~mm}$ were used in the DLS. The sample concentration was $1.0 \mathrm{mg} / \mathrm{mL}$. Size exclusion chromatography (SEC) measurements of poly(pentafluorophenyl acrylate) were performed on a Shimadzu Prominence-I LC-2030C 3D (Den Bosch, The Netherlands) with a Shimadzu RID-20A refractive index detector. THF was used as elution solvent with a flow of $1.0 \mathrm{~mL} / \mathrm{min}$ at an operating temperature of $40^{\circ} \mathrm{C}$. A mixed-C and mixed-D column in series (exclusion limit $=2,000,000 \mathrm{~g} / \mathrm{mol}$, $7.5 \mathrm{~mm}$ i.d. $\times 300 \mathrm{~m}$ ), calibrated using polystyrene (Agilent, Santa Clara, CA, USA), was used to determine the molecular weight. SEC measurements of the functionalized polymers were performed on a PL-GPC-50 plus from Polymer Laboratories (Varian Inc. Company, Palo Alto, CA, USA) with a refractive index detector. DMF with $10 \mathrm{mM} \mathrm{LiBr}$ was used as elution solvent with a flow of $1.0 \mathrm{~mL} / \mathrm{min}$ and at an operating temperature of $50{ }^{\circ} \mathrm{C}$. The implemented column was a Shodex GPC-KD-804 column (exclusion limit $=400,000 \mathrm{Da}$, $0.8 \mathrm{~cm}$ i.d. $\times 300 \mathrm{~mm}$ ), which has been calibrated with poly(ethylene oxide) (Polymer Laboratories). Dry solvents were obtained from MBraun solvent purification system (MB SPS-800, Stratham, NH, USA). To purify the functionalized polymers, standard RC Tubing from Spectrum Chemical (New Brunswick, NJ, USA) with a molecular weight cut-off (MWCO) of 6-8 $\mathrm{kDa}$ was used. All photocatalytic reactions were performed using 10 light-emitting diodes (LEDs) of Intelligent LED solution model ILH-XQ01-S380-SC211WIR200 (385 nm, 3.85 W UV LEDs, Thatcham, UK). Automated column chromatography was performed on a Biotage Isolera ${ }^{\circledR}$ One (Hengoed, UK) using a $150 \mathrm{~g}$ Biotage Silica cartridge.

\subsection{General Procedure for Photoredox Dehalogenation in Water}

A solution of $10 \mathrm{mg}$ of functionalized polymer in $0.5 \mathrm{~mL}$ of demineralized water was prepared. To this solution, the substrate $(50 \mu \mathrm{mol}, 1.0$ eq.), DIPEA $(43.7 \mu \mathrm{L}, 250 \mu \mathrm{mol}$, 5.0 eq.) and a magnetic stirrer were added. The sample was subsequently degassed for 5 min with argon. The top of the vial was wrapped with Parafilm to make the vial as airtight as possible. The degassed sample was placed in the irradiation set up and illuminated with $385 \mathrm{~nm}$ LED while being cooled with air for 3 or $18 \mathrm{~h}$, depending on the experiment. After the catalytic reaction, the sample was extracted 3 times with $0.5 \mathrm{~mL}$ of $\mathrm{CDCl}_{3}$. Conversion was then measured by ${ }^{1} \mathrm{H}$ NMR spectroscopy (estimated measurement error $\pm 2 \%$ ).

Synthesis of 10-(4-(2-((tert-butoxycarbonyl)amino)ethyl)phenyl)-3,6-di-tert-butyl-9-mesityl acridin-10-ium tetrafluoroborate (ACR- $\left.\mathbf{N H}_{2}\right)$. The synthesis was adapted from the literature [35]. 3,6-Di-tert-butyl-9-mesitylxanthylium tetrafluoroborate $(900 \mathrm{mg}, 1.8 \mathrm{mmol}$, 
1.0 eq.) was added to an oven dried flask and dry DCM (3.6 mL) was added. Subsequently, acetic acid (309.8 $\mu \mathrm{L}, 5.4 \mathrm{mmol}, 3.0$ eq.) and $\mathrm{NEt}_{3}(377.5 \mu \mathrm{L}, 2.7 \mathrm{mmol}, 1.5$ eq.) were added to the solution. tert-Butyl (4-aminophenethyl)carbamate $(853.4 \mathrm{mg}, 2.6 \mathrm{mmol}, 2 \mathrm{eq}$.) was dissolved separately in $2 \mathrm{~mL}$ of dry DCM and the solution was added dropwise to the reaction mixture. Next, the flask was wrapped in aluminum foil and the reaction mixture was stirred for $16 \mathrm{~h}$ at room temperature. The reaction mixture was subsequently washed with water $(1 \times 150 \mathrm{~mL})$ and brine $(1 \times 50 \mathrm{~mL})$. HBF 4 - $\mathrm{Et}_{2} \mathrm{O}$-complex $(220 \mu \mathrm{L}$, $1.8 \mathrm{mmol}, 1.0$ eq.) was added to the organic phase and swirled until homogeneity. The organic phase was washed with water $(1 \times 100 \mathrm{~mL})$ and $1 \mathrm{M}$ aq. $\mathrm{NaBF}_{4}(1 \times 100 \mathrm{~mL})$ and afterwards dried over $\mathrm{NaBF}_{4}$. After removing the solvent, the solid compound was triturated $3 \mathrm{x}$ with 1:2 $\mathrm{Et}_{2} \mathrm{O}$ :pentane and the obtained product was dried for $18 \mathrm{~h}$ in vacuo at room temperature. 10-(4-(2-((tert-butoxycarbonyl)-amino)ethyl)phenyl)-3,6-di-tert-butyl9-mesitylacridin-10-ium tetrafluoroborate $(1.144 \mathrm{~g}, 1.60 \mathrm{mmol}, 88 \%$ yield) was obtained as a dark yellow solid. ${ }^{1} \mathrm{H}$ NMR $\left(400 \mathrm{MHz}, \mathrm{CDCl}_{3}\right): \delta=7.83(\mathrm{t}, J=8.0 \mathrm{~Hz}, 2 \mathrm{H}), 7.79(\mathrm{~m}, 4 \mathrm{H})$, $7.61(\mathrm{~d}, J=8.0 \mathrm{~Hz}, 2 \mathrm{H}), 7.44(\mathrm{~s}, 2 \mathrm{H}), 7.17(\mathrm{~s}, 2 \mathrm{H}), 5.03(\mathrm{br}, 1 \mathrm{H}), 3.57(\mathrm{q}, J=8.0 \mathrm{~Hz}, 2 \mathrm{H})$, $3.14(\mathrm{t}, J=7.4 \mathrm{~Hz}, 2 \mathrm{H}), 2.49(\mathrm{~s}, 3 \mathrm{H}), 1.85(\mathrm{~s}, 6 \mathrm{H}), 1.30(\mathrm{~s}, 18 \mathrm{H}) \mathrm{ppm} .{ }^{13} \mathrm{C}$ NMR $(100 \mathrm{MHz}$, $\left.\mathrm{CDCl}_{3}\right): \delta=163.8,162.3,156.2,143.9,142.2,140.3,136.0,134.8,132.0,129.2,129.0,128.3$, $127.8,127.5,124.0,115.1,41.7,36.8,36.7,36.1,30.2,28.5,21.3,20.2$ ppm. ${ }^{19} \mathrm{~F}$ NMR $(376 \mathrm{MHz}$, $\mathrm{CDCl}_{3}: \delta=-153.7(\mathrm{~m}),-153.8(\mathrm{~m}) \mathrm{ppm}$. MALDI-TOF $\mathrm{m} / z$ calculated for $\mathrm{C}_{43} \mathrm{H}_{53} \mathrm{~N}_{2} \mathrm{O}_{2}$ $\left[\mathrm{M}-\mathrm{BF}_{4}\right]^{+}$629.41, found 629.43 .

10-(4-(2-((tert-Butoxycarbonyl)amino)ethyl)phenyl)-3,6-di-tert-butyl-9-mesitylacridin10-ium tetrafluoroborate $(250.0 \mathrm{mg}, 348.8 \mu \mathrm{mol}, 1.0$ eq.) was dissolved in $4 \mathrm{M} \mathrm{HCl}$ in dioxane $(5 \mathrm{~mL}, 17.4 \mathrm{mmol}, 50 \mathrm{eq}$.) and the solution was stirred at room temperature. After $6 \mathrm{~h}$, total conversion was confirmed by TLC (heptane:ethanol 4:1) and $5.5 \mathrm{~mL}$ of $4 \mathrm{M} \mathrm{NaOH}$ solution was added to the solution. The reaction mixture was stirred rigorously for $24 \mathrm{~h}$ at room temperature. Afterwards, the aqueous layer was extracted with DCM $(4 \times 25 \mathrm{~mL})$ and the combined organic layers were washed with water $(3 \times 25 \mathrm{~mL}) .1 \mathrm{M} \mathrm{NaBF}_{4}$ solution $(100 \mathrm{~mL})$ was added to the organic layer and stirred vigorously for $45 \mathrm{~min}$. The organic layer was separated from the aqueous phase and dried over $\mathrm{NaBF}_{4}$. After removing the solvent under reduced pressure, the solid compound was triturated $3 \mathrm{x}$ with 1:2 $\mathrm{Et}_{2} \mathrm{O}$ :pentane and dried for $18 \mathrm{~h}$ in vacuo at room temperature. 10-(4-(2-Aminoethyl)phenyl)-3,6-di-tert-butyl9-mesitylacridin-10-ium tetrafluoroborate ACR- $\mathbf{N H}_{2}(193.46 \mathrm{mg}, 313.76 \mu \mathrm{mol}, 90 \%$ yield) was obtained as bright yellow solid. ${ }^{1} \mathrm{H}$ NMR $\left(400 \mathrm{MHz}, \mathrm{CDCl}_{3}\right): \delta=8.01(\mathrm{~d}, J=8.1 \mathrm{~Hz}$, $2 \mathrm{H}), 7.78(\mathrm{~d}, J=1.9 \mathrm{~Hz}, 4 \mathrm{H}), 7.63-7.54(\mathrm{~m}, 2 \mathrm{H}), 7.47(\mathrm{~s}, 2 \mathrm{H}), 7.16(\mathrm{~s}, 2 \mathrm{H}), 6.88(\mathrm{~s}, 3 \mathrm{H}), 3.72(\mathrm{~d}$, $J=10.5 \mathrm{~Hz}, 2 \mathrm{H}), 3.58-3.49(\mathrm{~m}, 2 \mathrm{H}), 2.48(\mathrm{~s}, 3 \mathrm{H}), 1.83(\mathrm{~s}, 5 \mathrm{H}), 1.31(\mathrm{~s}, 18 \mathrm{H}) \mathrm{ppm} .{ }^{13} \mathrm{C} \mathrm{NMR}$ $\left(100 \mathrm{MHz}, \mathrm{CDCl}_{3}\right): \delta=163.3,161.3,141.1,140.5,139.3,134.9,134.2,131.7,128.2,128.0,127.2$, $126.9,126.7,122.9,114.2,40.6,35.8,31.9,29.2,21.3,20.3$ ppm. ${ }^{19} \mathrm{~F}$ NMR $\left(376 \mathrm{MHz}, \mathrm{CDCl}_{3}\right)$ : $\delta=-150.8(\mathrm{~d}, J=19.6 \mathrm{~Hz}) \mathrm{ppm}$. MALDI-TOF $m / z$ calculated for $\mathrm{C}_{38} \mathrm{H}_{45} \mathrm{~N}_{2}\left[\mathrm{M}-\mathrm{BF}_{4}\right]^{+} 529.36$ found 529.37 .

\section{Conclusions}

In this work, two reductive organic photocatalysts, namely 10-phenylphenothiazine and 9-mesityl-3,6-di-tert-butyl-10-phenyl-acridinium, were grafted to a polymeric backbone. The additional presence of hydrophobic and/or hydrophilic grafts, $n$-dodecylamine and Jeffamine, induced a hydrophobic collapse of the polymer in aqueous media, resulting in the formation of polymeric nanoparticles with hydrodynamic diameters $D_{H}$ of $11 \mathrm{~nm}$ and smaller. Owing to the customizability of the synthetic approach, six different types of amphiphilic polymers, each differing in either polymer backbone length or composition of functionalized groups, were readily accessible. These PNs were constructed in such a way that the effect of polymer microstructure-polymer length, catalyst loading and catalyst type - on catalyst activity and selectivity could be compared to one another.

All PNs were successful in the reduction of aryl halides in an aqueous medium upon irradiation with light of $385 \mathrm{~nm}$ for $3 \mathrm{~h}$. Due to the hydrophobic compartmentalization of these PNs, a high local substrate and catalyst concentration is obtained, resulting in shorter 
reaction times. As expected, phenothiazine, the less powerful but faster catalyst, showed higher conversions compared to acridinium (at equal catalyst loading) for substrates that had a lower reduction potential. The catalytic activity of the phenothiazine, however, dropped quickly when more resilient aryl halides were used as substrates. The PNs with a higher catalyst loading, which had no additional $n$-dodecyl grafts, showed the highest conversion for most substrates. Altering the length of the polymeric backbone did not show any clear difference in catalytic activity.

Finally, the PNs were used to perform a C-C cross-coupling reaction between 4chlorobenzonitrile and $N$-methylpyrrole that competes with the corresponding reduction reaction. Due to the high local concentration of the substrate within the nanoparticle, shorter reaction times and significantly less $N$-methylpyrrole were necessary to achieve reasonable selectivity towards the $\mathrm{C}-\mathrm{C}$ cross-coupled product. The results show that attaching organic photocatalysts to amphiphilic polymers is an effective way to create tailor-made nanoreactors for efficient catalysis in water. By using these PNs, shorter reaction times and less substrate are needed to reach a high conversion. This, together with the fact that these photocatalysts are light driven, makes the use of these PNs an effective and sustainable approach to perform photoredox catalysis in aqueous media.

Supplementary Materials: The following are available online, Figure S1: SEC traces; Figures S2-S7: NMR spectra for polymer functionalization; Figure S8: SEC traces; Figure S9: DLS results; Figure S10: UV/Vis spectra; Figure S11: Irradiation setup; Figures S12-S13: Reaction mechanisms; Figures S14-S24: NMR spectra for catalysis experiments; Figures S25-S48: NMR spectra for compound characterization.

Author Contributions: F.E., T.H.R.K. and D.v.K. synthesized, isolated, and characterized the compounds; D.v.K. constructed the irradiation setup; T.H.R.K. and D.v.K. performed catalysis experiments and T.H.R.K. analyzed substrate conversion by NMR spectroscopy; F.E. and A.R.A.P. designed the study; F.E., T.H.R.K. and A.R.A.P. wrote and edited the manuscript. All authors have read and agreed to the published version of the manuscript.

Funding: F.E. acknowledges the Alexander von Humboldt Foundation for providing a Feodor Lynen research fellowship.

Institutional Review Board Statement: Not applicable.

Informed Consent Statement: Not applicable.

Data Availability Statement: All data are available from the corresponding author upon request.

Conflicts of Interest: The authors declare no conflict of interest.

Sample Availability: Samples of the compounds PN1-6 are available from the authors.

\section{References}

1. Narayanam, J.M.R.; Stephenson, C.R.J. Visible light photoredox catalysis: Applications in organic synthesis. Chem. Soc. Rev. 2011, 40, 102-113. [CrossRef] [PubMed]

2. Corrigan, N.; Shanmugam, S.; Xu, J.; Boyer, C. Photocatalysis in organic and polymer synthesis. Chem. Soc. Rev. 2016, 45, 6165-6212. [CrossRef] [PubMed]

3. Glaser, F.; Wenger, O.S. Recent progress in the development of transition-metal based photoredox catalysts. Coord. Chem. Rev. 2020, 405, 213129. [CrossRef]

4. Prier, C.K.; Rankic, D.A.; MacMillan, D.W.C. Visible light photoredox catalysis with transition metal complexes: Applications in organic synthesis. Chem. Rev. 2013, 113, 5322-5363. [CrossRef] [PubMed]

5. Cannalire, R.; Pelliccia, S.; Sancineto, L.; Novellino, E.; Tron, G.C.; Giustiniano, M. Visible light photocatalysis in the late-stage functionalization of pharmaceutically relevant compounds. Chem. Soc. Rev. 2021, 50, 866-897. [CrossRef]

6. Giedyk, M.; Narobe, R.; Weiß, S.; Touraud, D.; Kunz, W.; König, B. Photocatalytic activation of alkyl chlorides by assemblypromoted single electron transfer in microheterogeneous solutions. Nat. Catal. 2020, 3, 40-47. [CrossRef]

7. Naumann, R.; Goez, M. A Green-LED Driven Source of Hydrated Electrons Characterized from Microseconds to Hours and Applied to Cross-Couplings. Chem. Eur. J. 2018, 24, 9833-9840. [CrossRef]

8. Bu, M.; Cai, C.; Gallou, F.; Lipshutz, B.H. PQS-enabled visible-light iridium photoredox catalysis in water at room temperature. Green Chem. 2018, 20, 1233-1237. [CrossRef]

9. Guerrero, I.; Kelemen, Z.; Viñas, C.; Romero, I.; Teixidor, F. Metallacarboranes as Photoredox Catalysts in Water. Chem. Eur. J. 2020, 26, 5027-5036. [CrossRef] 
10. Naumann, R.; Goez, M. How the sustainable solvent water unleashes the photoredox catalytic potential of ruthenium polypyridyl complexes for pinacol couplings. Green Chem. 2019, 21, 4470-4474. [CrossRef]

11. Bobo, M.V.; Kuchta, J.J.; Vannucci, A.K. Recent advancements in the development of molecular organic photocatalysts. Org. Biomol. Chem. 2021, 19, 4816-4834. [CrossRef] [PubMed]

12. Romero, N.A.; Nicewicz, D.A. Organic Photoredox Catalysis. Chem. Rev. 2016, 116, 10075-10166. [CrossRef]

13. Lee, Y.; Kwon, M.S. Emerging Organic Photoredox Catalysts for Organic Transformations. Eur. J. Org. Chem. 2020, 2020, 6028-6043. [CrossRef]

14. Discekici, E.H.; Treat, N.J.; Poelma, S.O.; Mattson, K.M.; Hudson, Z.M.; Luo, Y.; Hawker, C.J.; de Alaniz, J.R. A highly reducing metal-free photoredox catalyst: Design and application in radical dehalogenations. Chem. Commun. 2015, 51, 11705-11708 [CrossRef] [PubMed]

15. Poelma, S.O.; Burnett, G.L.; Discekici, E.H.; Mattson, K.M.; Treat, N.J.; Luo, Y.; Hudson, Z.M.; Shankel, S.L.; Clark, P.G.; Kramer, J.W.; et al. Chemoselective Radical Dehalogenation and C-C Bond Formation on Aryl Halide Substrates Using Organic Photoredox Catalysts. J. Org. Chem. 2016, 81, 7155-7160. [CrossRef] [PubMed]

16. MacKenzie, I.A.; Wang, L.; Onuska, N.P.R.; Williams, O.F.; Begam, K.; Moran, A.M.; Dunietz, B.D.; Nicewicz, D.A. Discovery and characterization of an acridine radical photoreductant. Nature 2020, 580, 76-80. [CrossRef] [PubMed]

17. Kerzig, C.; Goez, M. Combining energy and electron transfer in a supramolecular environment for the "green" generation and utilization of hydrated electrons through photoredox catalysis. Chem. Sci. 2016, 7, 3862-3868. [CrossRef]

18. Naumann, R.; Lehmann, F.; Goez, M. Generating Hydrated Electrons for Chemical Syntheses by Using a Green Light-Emitting Diode (LED). Angew. Chem. Int. Ed. 2018, 57, 1078-1081. [CrossRef]

19. Pallini, F.; Sangalli, E.; Sassi, M.; Roth, P.M.C.C.; Mattiello, S.; Beverina, L. Selective photoredox direct arylations of aryl bromides in water in a microfluidic reactor. Org. Biomol. Chem. 2021, 19, 3016-3023. [CrossRef]

20. Bu, M.; Lu, G.; Jiang, J.; Cai, C. Merging visible-light photoredox and micellar catalysis: Arylation reactions with anilines nitrosated in situ. Catal. Sci. Technol. 2018, 8, 3728-3732. [CrossRef]

21. Finck, L.; Brals, J.; Pavuluri, B.; Gallou, F.; Handa, S. Micelle-Enabled Photoassisted Selective Oxyhalogenation of Alkynes in Water under Mild Conditions. J. Org. Chem. 2018, 83, 7366-7372. [CrossRef]

22. Xie, H.-Y.; Han, L.-S.; Huang, S.; Lei, X.; Cheng, Y.; Zhao, W.; Sun, H.; Wen, X.; Xu, Q.-L. N-Substituted 3(10 H)-Acridones as Visible-Light, Water-Soluble Photocatalysts: Aerobic Oxidative Hydroxylation of Arylboronic Acids. J. Org. Chem. 2017, 82, 5236-5241. [CrossRef]

23. Eisenreich, F.; Meijer, E.W.; Palmans, A.R.A. Amphiphilic Polymeric Nanoparticles for Photoredox Catalysis in Water. Chem. Eur. J. 2020, 26, 10355-10361. [CrossRef]

24. Lipshutz, B.H.; Ghorai, S.; Cortes-Clerget, M. The Hydrophobic Effect Applied to Organic Synthesis: Recent Synthetic Chemistry "in Water". Chem. Eur. J. 2018, 1-25. [CrossRef]

25. Lipshutz, B.H. Synthetic chemistry in a water world. New rules ripe for discovery. Curr. Opin. Green Sustain. Chem. 2018, 11, 1-8. [CrossRef]

26. Bergbreiter, D.E.; Liu, Y.-S. Amphoteric, water-soluble polymer-bound hydrogenation catalysts. Tetrahedron Lett. 1997, 38, 3703-3706. [CrossRef]

27. Liang, Y.; Bergbreiter, D.E. Recyclable polyisobutylene (PIB)-bound organic photoredox catalyst catalyzed polymerization reactions. Polym. Chem. 2016, 7, 2161-2165. [CrossRef]

28. Bergbreiter, D.E. Soluble polymers as tools in catalysis. ACS Macro Lett. 2014, 3, 260-265. [CrossRef]

29. Treat, N.J.; Sprafke, H.; Kramer, J.W.; Clark, P.G.; Barton, B.E.; Read De Alaniz, J.; Fors, B.P.; Hawker, C.J. Metal-Free Atom Transfer Radical Polymerization. J. Am. Chem. Soc. 2014, 136, 16096-16101. [CrossRef]

30. Terashima, T.; Mes, T.; de Greef, T.F.A.; Gillissen, M.A.J.; Besenius, P.; Palmans, A.R.A.; Meijer, E.W. Single-chain folding of polymers for catalytic systems in water. J. Am. Chem. Soc. 2011, 133, 4742-4745. [CrossRef]

31. Artar, M.; Souren, E.R.J.; Terashima, T.; Meijer, E.W.; Palmans, A.R.A. Single Chain Polymeric Nanoparticles as Selective Hydrophobic Reaction Spaces in Water. ACS Macro Lett. 2015, 4, 1099-1103. [CrossRef]

32. Huerta, E.; Stals, P.J.M.; Meijer, E.W.; Palmans, A.R.A. Consequences of Folding a Water-Soluble Polymer around an Organocatalyst. Angew. Chem. Int. Ed. 2013, 52, 2906-2910. [CrossRef] [PubMed]

33. Liu, Y.; Turunen, P.; de Waal, B.F.M.M.; Blank, K.G.; Rowan, A.E.; Palmans, A.R.A.; Meijer, E.W. Catalytic single-chain polymeric nanoparticles at work: From ensemble towards single-particle kinetics. Mol. Syst. Des. Eng. 2018, 3, 609-618. [CrossRef]

34. Yan, J.; Pan, X.; Schmitt, M.; Wang, Z.; Bockstaller, M.R.; Matyjaszewski, K. Enhancing Initiation Efficiency in Metal-Free Surface-Initiated Atom Transfer Radical Polymerization (SI-ATRP). ACS Macro Lett. 2016, 5, 661-665. [CrossRef]

35. White, A.; Wang, L.; Nicewicz, D. Synthesis and Characterization of Acridinium Dyes for Photoredox Catalysis. Synlett 2019, 30, 827-832. [CrossRef]

36. Zilate, B.; Fischer, C.; Sparr, C. Design and application of aminoacridinium organophotoredox catalysts. Chem. Commun. 2020, 56, 1767-1775. [CrossRef]

37. Timpe, H.-J.; Ulrich, S.; Decker, C.; Fouassier, J.-P. Acridinium salt mediated photopolymerization of acrylates and methacrylates with dihydropyridine-iodonium salt combinations. Eur. Polym. J. 1994, 30, 1301-1307. [CrossRef]

38. Theato, P. Synthesis of well-defined polymeric activated esters. J. Polym. Sci. Part A Polym. Chem. 2008, 46, 6677-6687. [CrossRef] 
39. Liu, Y.; Pauloehrl, T.; Presolski, S.I.; Albertazzi, L.; Palmans, A.R.A.; Meijer, E.W. Modular Synthetic Platform for the Construction of Functional Single-Chain Polymeric Nanoparticles: From Aqueous Catalysis to Photosensitization. J. Am. Chem. Soc. 2015, 137, 13096-13106. [CrossRef]

40. ter Huurne, G.M.; de Windt, L.N.J.; Liu, Y.; Meijer, E.W.; Voets, I.K.; Palmans, A.R.A. Improving the Folding of Supramolecular Copolymers by Controlling the Assembly Pathway Complexity. Macromolecules 2017, 50, 8562-8569. [CrossRef]

41. Yamamoto, H.; Morishima, Y. Effect of hydrophobe content on intra- and interpolymer self-associations of hydrophobically modified poly(sodium 2-(acrylamido)-2-methylpropanesulfonate) in water. Macromolecules 1999, 32, 7469-7475. [CrossRef]

42. Morishima, Y.; Nomura, S.; Ikeda, T.; Seki, M.; Kamachi, M. Characterization of Unimolecular Micelles of Random Copolymers of Sodium 2-(Acrylamido)-2-methylpropanesulfonate and Methacrylamides Bearing Bulky Hydrophobic Substituents. Macromolecules 1995, 28, 2874-2881. [CrossRef]

43. Steiner, A.; Williams, J.D.; Rincón, J.A.; de Frutos, O.; Mateos, C.; Kappe, C.O. Implementing Hydrogen Atom Transfer (HAT) Catalysis for Rapid and Selective Reductive Photoredox Transformations in Continuous Flow. Eur. J. Org. Chem. 2019, 2019, 5807-5811. [CrossRef]

44. Connell, T.U.; Fraser, C.L.; Czyz, M.L.; Smith, Z.M.; Hayne, D.J.; Doeven, E.H.; Agugiaro, J.; Wilson, D.J.D.; Adcock, J.L.; Scully, A.D.; et al. The Tandem Photoredox Catalysis Mechanism of [ $\operatorname{Ir}(\mathrm{ppy}) 2$ (dtb-bpy)] + Enabling Access to Energy Demanding Organic Substrates. J. Am. Chem. Soc. 2019, 141, 17646-17658. [CrossRef] [PubMed]

45. Yang, W.; You, D.; Li, C.; Han, C.; Tang, N.; Yang, H.; Xue, X. Photolysis of Nitroaromatic Compounds under Sunlight: A Possible Daytime Photochemical Source of Nitrous Acid? Environ. Sci. Technol. Lett. 2021, 8, 747-752. [CrossRef]

46. Cowper, N.G.W.; Chernowsky, C.P.; Williams, O.P.; Wickens, Z.K. Potent Reductants via Electron-Primed Photoredox Catalysis: Unlocking Aryl Chlorides for Radical Coupling. J. Am. Chem. Soc. 2020, 142, 2093-2099. [CrossRef] [PubMed]

47. Fry, A.J.; Krieger, R.L. Electrolyte effects upon the polarographic reduction of alkyl halides in dimethyl sulfoxide. J. Org. Chem. 1976, 41, 54-57. [CrossRef]

48. Takeda, N.; Poliakov, P.V.; Cook, A.R.; Miller, J.R. Faster Dissociation: Measured Rates and Computed Effects on Barriers in Aryl Halide Radical Anions. J. Am. Chem. Soc. 2004, 126, 4301-4309. [CrossRef]

49. Cranwell, P.; Russell, A.; Smith, C. Methyl Hydrazinocarboxylate as a Practical Alternative to Hydrazine in the Wolff-Kishner Reaction. Synlett 2015, 27, 131-135. [CrossRef] 